\title{
Surgical wound infection following open humeral fracture caused by Mycobacterium houstonense: a case report
}

\author{
Lei Tian', Zhen Zhang ${ }^{2}$, Zhongju Chen ${ }^{1}$ and Ziyong Sun ${ }^{1 *}$
}

\begin{abstract}
Background: Historically Mycobacterium houstonense belongs to the unnamed third biovariant complex of the Mycobacterium fortuitum group, which are sorbitol positive. To date, there have been few reports of human infection induced by $M$. houstonense worldwide.

Case presentation: We describe the case of a 68-year-old man with surgical wound infection, following an open humeral fracture, caused by M. houstonense and Escherichia coli. An implant bone plate had been embedded for internal fixation during surgery on the humeral fracture previously. A week later $E$. coli was isolated from the skin wound secretions. Cefoperazone-sulbactam was used for treatment for two weeks but the infection was not controlled, with a subsequent risk of deep wound infection. External fixation of the fracture was then performed instead of internal fixation. Ten days later, M. houstonense was isolated from new wound secretions. M. houstonense was identified by the molecular sequencing method. The TREK Diagnostic System was used to test the susceptibility to antibiotics by the microbroth dilution method. Levofloxacin and amikacin were used for treatment according to the results of the susceptibility test and the patient's condition obviously improved.
\end{abstract}

Conclusion: To the best of our knowledge, this is the first case in China of human surgical wound infection caused by $M$. houstonense following open humeral fracture. The combination of levofloxacin and amikacin was effective in the treatment of $M$. houstonense infection.

Keywords: Mycobacteria houstonense, Mycobacterium fortuitum group, TREK diagnostic systems, Microbroth dilution method, Escherichia coli

\section{Background}

Rapidly growing mycobacteria (RGM) can produce colonies that do not appear or slowly appear with pigmentation on various solid media within 7 days (most within 3 to 4 days), and contain mycobacterial acid and maintain amylase activity for 3 days or 2 weeks [1, 2]. Traditional classification methods relying on biochemical and phenotypic identification are labor intensive. However, new technologies such as high-performance liquid chromatography, 16S rRNA gene sequencing and PCR restriction fragment length polymorphism analysis have been more recently adopted [1]. The species of RGM capable of

\footnotetext{
* Correspondence: zysun@tjh.tjmu.edu.cn

${ }^{1}$ Department of Clinical Laboratory, Tongji Hospital, Tongji Medical College, Huazhong University of Science and Technology, Wuhan, Hubei Province, China

Full list of author information is available at the end of the article
}

producing disease in humans consist primarily of the Mycobacterium fortuitum group, the Mycobacterium chelonae/abscessus group and the Mycobacterium smegmatis group [1]. Historically, the M. fortuitum group comprised M. fortuitum (formerly M. fortuitum biovar fortuitum), Mycobacterium peregrinum (formerly $M$. fortuitum biovar peregrinum) and the taxon known as the unnamed third biovariant complex. The $M$. fortuitum third biovariant complex mainly included sorbitol positive and sorbitol negative organisms, represented by Mycobacterium houstonense and Mycobacterium bonickei, respectively.

At present, based on the evolution of $16 \mathrm{~S}$ ribosomal gene (rDNA) sequencing, the unnamed third biovariant complex includes the Mycobacterium species: M. senegalense, M. mageritense, M. septicum, M. porcinum, $M$. neworleanense, $M$. brisbanense, $M$. houstonense and $M$. bonickei $[1,3,4]$. Among the third biovariant complex, 
their $16 \mathrm{~S}$ rDNA sequences generally differ by $15 \mathrm{bp}$ or fewer [1].

M. houstonense was first reported by the CDC (USA) and was isolated from the face wound of a patient who lived in Houston, Texas, so was named Mycobacterium houston $[4,5]$. To date, almost all of the reports of the now-called $M$. houstonense have been in the USA and Australia [1]. Here we report an infectious case of $M$. houstonense in China. To the best of our knowledge this is the first report of $M$. houstonense in China.

\section{Case presentation}

A 68-year-old man had an open fracture of the right humerus due to a fall. The patient was sent to the hospital as an emergency case. The doctor performed debridement and suture of the patient's wound. When all of the test indexes were normal, surgery of the humeral fracture was undertaken. Large bone defects in the middle and lower parts of the humerus were found during the operation. (Fig. 1) After proper shortening of the fracture end, a bone plate implant was embedded for internal fixation. (Fig. 2).

A week later, wound secretions exuded through the original drainage tube. Escherichia coli was isolated from the wound secretion by culture. E. coli isolates were multidrug resistant as determined by antimicrobial susceptibility testing using the disk diffusion test. The

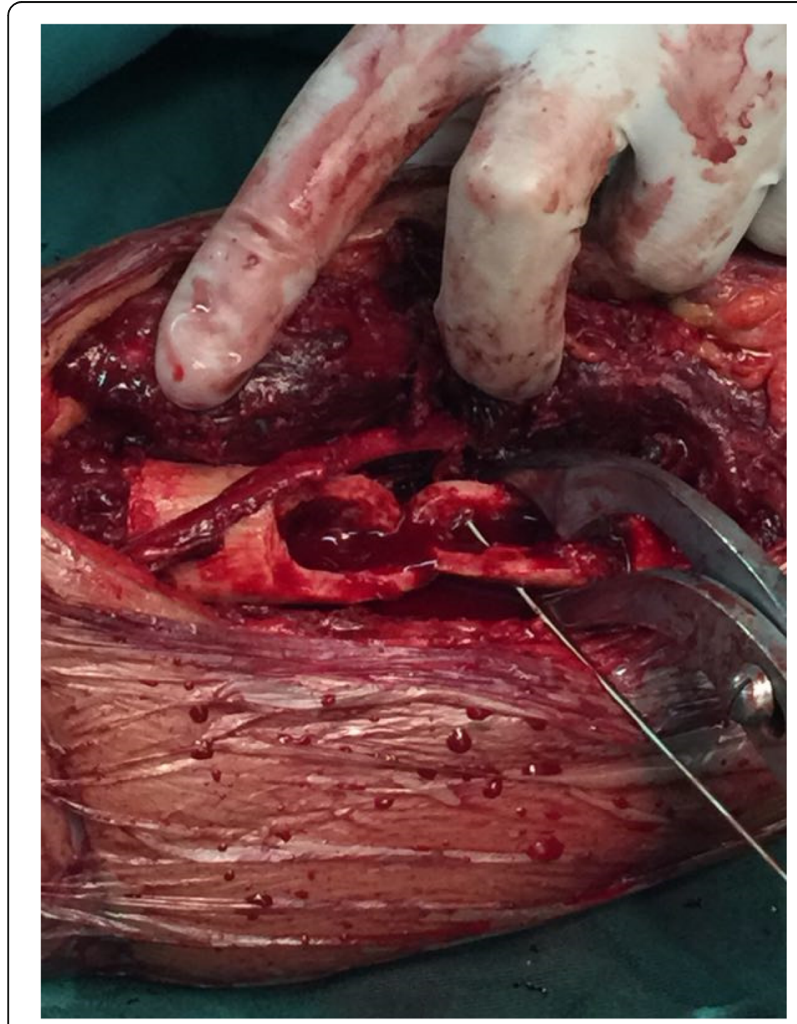

Fig. 1 Large bone defects in the middle and lower part of the humerus

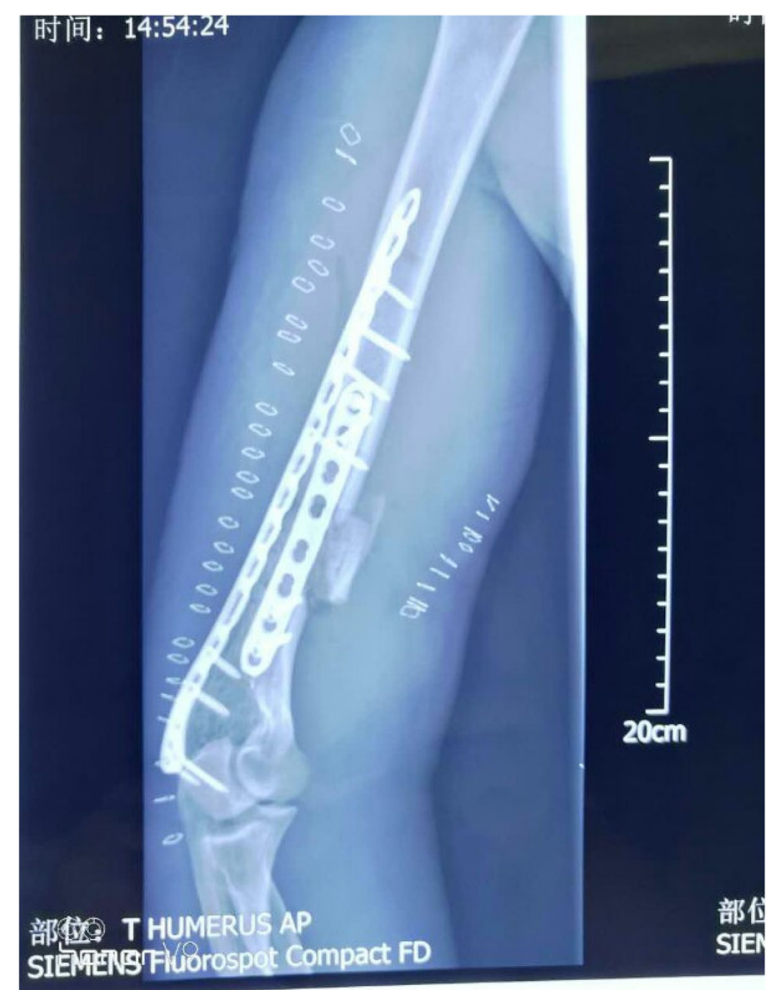

Fig. 2 Implant bone plate was embedded for internal fixation

procedure and interpretation of the results of the antimicrobial susceptibility tests were conducted in accordance with the CLSI 2018 guidelines [6]. Antimicrobial drugs and Mueller-Hinton media for the disk diffusion test were obtained from Oxoid Company, UK. The results showed that the strain was resistant to cefazolin, cefotaxime, cefepime, aztreonam, ampicillin, piperacillin, ciprofloxacin, levofloxacin, moxifloxacin, chloramphenicol, tetracycline and trimethoprim/sulfamethoxazole, but sensitive to gentamicin, amikacin, imipenem, meropenem, ceftazidime, amoxicillin/clavulanate, piperacillin/ tazobactam, cefoperazone/sulbactam and cefoxitin. Negative pressure attraction was performed with a progressive artificial skin cover and cefoperazone/sulbactam was used for treatment. Cefoperazone/sulbactam, which combined cefoperazone $(2000 \mathrm{mg})$ with sulbactam $(1000 \mathrm{mg})$, was used via intravenous infusion, once every $12 \mathrm{~h}$.

Two weeks later, the drainage tube had been closed but yellowish cloudy secretions exuded on the lateral side of the arm incision. The doctors suspected that the deep wound was infected, therefore, re-debridement of the patient's wound and external fixation of the fracture were performed (Fig. 3).

Ten days later, another secretion from the wound was observed (Fig. 4). RGM were isolated from the secretion by culture and were identified as belonging to the $M$. fortuitum group using an IVD-MALDIBIOTYPER (Bruker, 


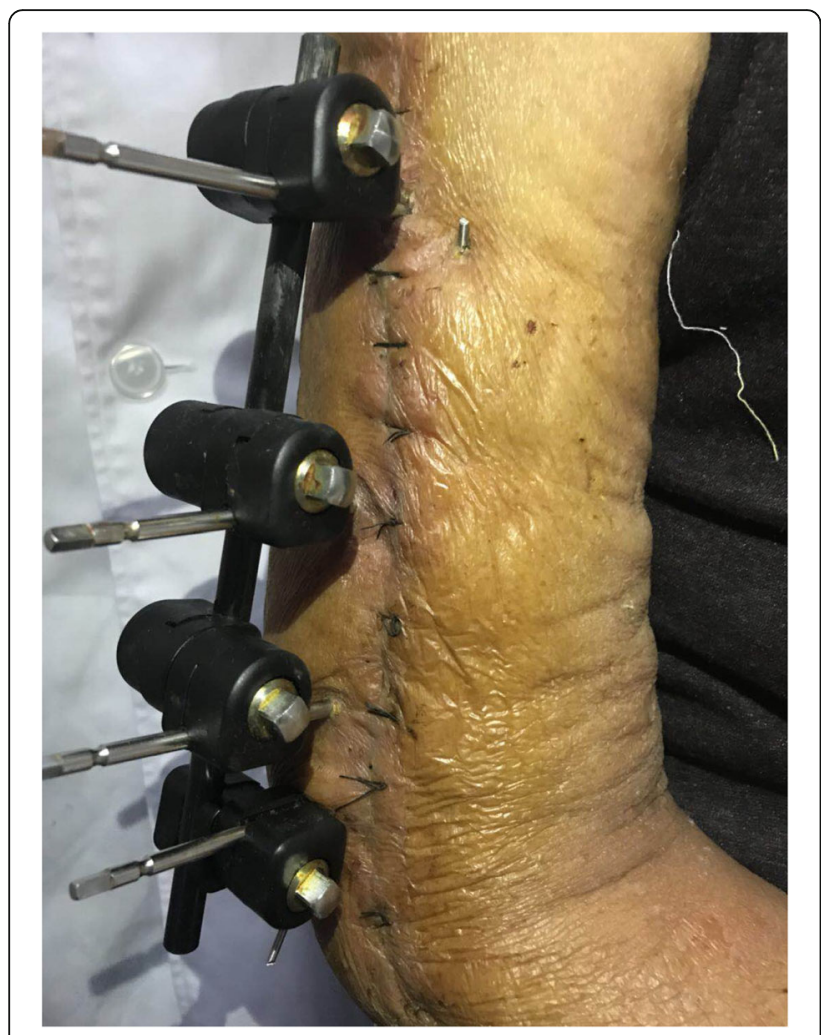

Fig. 3 External fixation of fracture were done

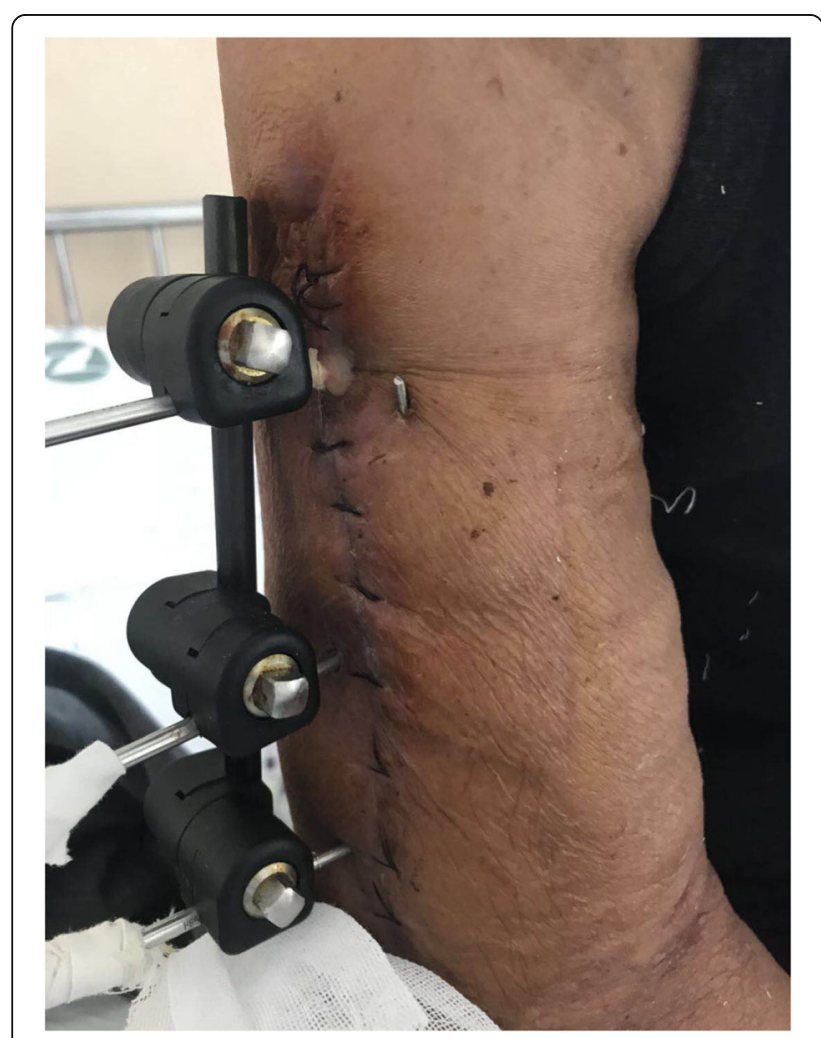

Fig. 4 Secretion was seen in the wound
Karlsruhe, Germany). The isolated strain was identified as $M$. houstonense by sequencing analysis. Monoclonal colonies were scraped and genomic DNA of the isolate was extracted using a commercial kit (DNeasy Blood and Tissue Kit; Qiagen, Germany). Primer design was based on the reports of Lane(1991) and CLSI MM18-A, and the primers for 16S rRNA PCR were as follows 27F: AGAGTTTGA TMTGGCTCAG, 1492R: TACGGYTACCTTGTTACGAC TT. The amplification conditions for PCR were based on those of previous reports [7, 8], and a PCR cycler (PTC220, Bio-Rad, USA) and first generation sequencer (Life Technology 2500 DX, ABI, Japan) were used. The amplified products were determined by comparing their restriction patterns with those available in the National Center for Biotechnology Information GenBank database. The results revealed sequence similarity (above 98.58\%) with $M$. houstonense (GenBank accession no. NR_042913.1).

The TREK Diagnostic System (Thermo, Germany) was used to test the antimicrobial drug susceptibility of $M$. houstonense by the microbroth dilution method. The antimicrobial drug sensitivity results were interpreted according to the CLSI M24 A2 guidelines [9]. The results, detailed in Table 1 , showed that $M$. houstonense was only sensitive to levofloxacin, moxifloxacin and amikacin.

A daily intravenous drip of $0.3 \mathrm{~g}$ of levofloxacin and injection of $100 \mathrm{ml}$ of sodium chloride, and injection of $0.2 \mathrm{~g}$ of amikacin and $250 \mathrm{ml}$ of sodium chloride twice a day, were used for treatment. Three weeks later, the wound was healing well and no secretions were detected.

\section{Discussion}

To the best of our knowledge this is the first report of $M$. houstonense in China. Reports of infection induced by $M$. houstonense are scarce throughout the world. The mechanism of infection with this organism remains unclear. Some studies have reported the isolation of $M$. houstonense from freshwater fish and fish products, especially retail frozen fish [10]. Freshwater fish might therefore be a reservoir of $M$. houstonense. However, in this case, the patient had not recently been exposed to fish products. In the first report of $M$. houstonense in the USA, M. houstonense was isolated from a wound in the patient's face $[4,5]$. Similar to many other RGM, $M$. houstonense often inhabits water, soil and dust. Healthcare-associated outbreaks and community-acquired diseases of RGM are usually related to the pollution of water or soil [1]. Stepping on a nail, motor vehicle accidents and compound fractures are typical of the clinical histories seen in patients with RGM infections [11]. In this case, the patient became infected with $M$. houstonense after an open fracture. 
Table 1 Antimicrobial sensitivity of Mycobacterium houstonense

\begin{tabular}{|c|c|c|c|c|c|}
\hline \multirow[t]{2}{*}{ Antibacterial drugs } & \multirow{2}{*}{$\begin{array}{l}\text { MIC } \\
(\mathrm{ug} / \mathrm{ml})\end{array}$} & \multicolumn{3}{|c|}{ Breakpoints $(\mu \mathrm{g} / \mathrm{mL})$} & \multirow[t]{2}{*}{ Results } \\
\hline & & Susceptible & Intermediate & Resistant & \\
\hline trimethoprim sulfamethoxazole & $4 / 76$ & $\leq 2 / 38$ & - & $\geq 4 / 76$ & resistant \\
\hline ciprofloxacin & 1 & $\leq 1$ & 2 & $\geq 4$ & sensitive \\
\hline moxifloxacin & $\leq 0.25$ & $\leq 1$ & 2 & $\geq 4$ & sensitive \\
\hline cefoxitin & $\geq 128$ & $\leq 16$ & $32-64$ & $\geq 128$ & resistant \\
\hline amikacin & 4 & $\leq 16$ & 32 & $\geq 64$ & sensitive \\
\hline doxycycline & $\geq 16$ & $\leq 1$ & $2-4$ & $\geq 8$ & resistant \\
\hline tegafycline & 0.12 & - & - & - & - \\
\hline linezolid & 32 & $\leq 8$ & 16 & $\geq 32$ & resistant \\
\hline imipenem & 32 & $\leq 4$ & $8-16$ & $\geq 32$ & resistant \\
\hline cefepime & $\geq 32$ & - & - & - & - \\
\hline amoxicillin-clavulanate & $32 / 16$ & - & - & - & - \\
\hline cefatriaxone & $\geq 64$ & - & - & - & - \\
\hline minocycline & 4 & - & - & - & - \\
\hline tobramycin & 8 & $\leq 2$ & 4 & $\geq 8$ & resistant \\
\hline clarithromycin & $\geq 16$ & $\leq 2$ & 4 & $\geq 8$ & resistant \\
\hline
\end{tabular}

Note: - No Breakpoint in CLSI M24-A2

RGM are widely distributed and human infections due to RGM have been reported in most geographic areas of the world [1], with the exception of $M$. senegalense that was originally found in Africa and has never been described elsewhere [12]. According to the limited literature, human infections induced by $M$. houstonense have mostly been reported in the USA and Australia to date. This is the first study to report the existence of $M$. houstonense in China, widening the regional distribution of $M$. houstonense throughout the world.

Many experimental methods have been reported for drug susceptibility tests of RGM, such as agar disk elution, the E-test, broth microdilution and agar disk diffusion. Each method has its own advantages and disadvantages. The microbroth dilution method is recommended by the CLSI as a RGM drug sensitivity test, and is currently considered the gold standard. In the CLSI M24 A2 guidelines, the break points of the MIC method for amikacin, tobramycin, cefoxitin, clarithromycin, ciprofloxacin, moxifloxacin, doxycycline, imipenem, meropenem, linezolid and trimethoprim/sulfamethoxazole were specified [9]. In this study, a drug susceptibility test strip of the TREK Diagnostic System (Thermo Company) was used. Besides the 11 drugs mentioned above, cefepime, amoxicillin-clavulanate, cefatriaxone and minocycline were also tested, but no break points were available for the interpretation of these four drugs.

Little is known about potential antimicrobial therapies to treat $M$. houstonense infections. For the treatment of RGM, therapeutic regimens frequently vary depending on the nature of the disease. Single drug therapy for small or localized lesions is usually effective. However, in the case of diffuse or pulmonary infections, it is necessary to combine oral and injectable antibiotics [1]. A report analyzing the $M$. fortuitum group showed $>90 \%$ susceptibility or intermediate susceptibility to amikacin, cefoxitin, ciprofloxacin, gatifloxacin, imipenem, levofloxacin, linezolid, sulfamethoxazole or trimethoprimsulfamethoxazole, and $<90 \%$ susceptibility or intermediate susceptibility to clarithromycin, doxycycline and vancomycin [1]. As previously reported, M. houstonense isolate 14,133 appeared susceptible to clarithromycin (MIC < $4 \mu \mathrm{g} / \mathrm{ml}$ ), but overnight incubation in clarithromycin $(0.1 \mu \mathrm{g} / \mathrm{ml})$ increased the clarithromycin MIC to $>128 \mu \mathrm{g} /$ $\mathrm{ml}[1,13]$. The clarithromycin MIC remained at $\leq 2 \mu \mathrm{g} / \mathrm{ml}$ for organisms incubated in the absence of macrolides $[1$, 13]. These results indicated that erm genes are inducible at the molecular level. Therefore, it is necessary to be careful when interpreting the drug sensitivity of macrolides. M. houstonense is known to express the intrinsic macrolide erm gene, so inducible drug resistance may occur affecting the macrolide susceptibility results $[5,13]$. Our drug susceptibility test showed that $M$. houstonense was only susceptible to levofloxacin, moxifloxacin and amikacin. In this case, the patient was treated with a combination of levofloxacin and amikacin intravenous drip according to the drug sensitivity results. The therapeutic effect was good. However, a standardized treatment for $M$. houstonense remains to be proposed.

A slight wound infection induced by RGM can heal itself and minor wound infections can be resolved be surgical debridement [1]. Many cases of extrapulmonary 
infections have been reported long before they were cured by surgical debridement alone, but these cases are prone to recurrence within 4-6 weeks [1, 14]. Surgical debridement combined with appropriate antimicrobial therapy can effectively avoid the recurrence of infection [11, 14-16]. In this case, surgical debridement combined with antimicrobial therapy based on the results of drug susceptibility tests was effective in the treatment of $M$. houstonense.

\section{Conclusion}

This case report describes an open fracture infection caused by combined infection of E. coli and M. houstonense. As the first report of infection caused by $M$. houstonense in China, it aids our understanding of the distribution of $M$. houstonense throughout the world.

\section{Abbreviation}

RGM: Rapidly growing mycobacreria

\section{Acknowledgements}

We thank Rui Zhu for his help of providing the patient's previous condition We are particularly grateful to THERMO corporation for providing the antimicrobial sensitivity test strip (TREK Diagnostic Systems,SENSITITRE) for this experiment. We thank Kate Fox, DPhil, from Liwen Bianji, Edanz Group China (www.liwenbianji.cn/ac), for editing the English text of a draft of this manuscript.

\section{Funding}

This work was supported by research grants from the National Mega Project on Major Infectious Disease Prevention (2017ZX10103005-007).

\section{Availability of data and materials}

All data is contained within the manuscript. Clinical isolates will be made available upon requests from Dr. Ziyong Sun.

\section{Authors' contributions}

LT drafted the manuscript. ZZ helped and revised the manuscript. ZJC and ZYS were in charge of the laboratory work. All authors read and approved the final manuscript.

\section{Ethics approval and consent to participate}

The study protocol was approved by the Tongji Hospital ethics committee for research in health. Informed written consent was obtained from the patient.

\section{Consent for publication}

The patient provided written consent for the case details to be published.

\section{Competing interests}

The authors declare that they have no competing interest.

\section{Publisher's Note}

Springer Nature remains neutral with regard to jurisdictional claims in published maps and institutional affiliations.

\section{Author details}

'Department of Clinical Laboratory, Tongji Hospital, Tongji Medical College, Huazhong University of Science and Technology, Wuhan, Hubei Province, China. ${ }^{2}$ Department of Pharmacy, Tongji Hospital, Tongji Medical College, Huazhong University of Science and Technology, Wuhan, Hubei Province, China.
Received: 20 December 2017 Accepted: 11 April 2019

Published online: 23 April 2019

\section{References}

1. Brown-Elliott BA, Wallace RJ Jr. Clinical and taxonomic status of pathogenic nonpigmented or late-pigmenting rapidly growing mycobacteria. Clin Microbiol Rev. 2002;15(4):716-46.

2. Wayne $L G$, Sramek HA. Agents of newly recognized or infrequently encountered mycobacterial diseases. Clin Microbiol Rev. 1992:5(1):1-25.

3. Adekambi T, Colson P, Drancourt M. rpoB-based identification of nonpigmented and late-pigmenting rapidly growing mycobacteria. J Clin Microbiol. 2003:41(12): 5699-708.

4. Schinsky MF, Morey RE, Steigerwalt AG, Douglas MP, Wilson RW, Floyd MM, Butler WR, Daneshvar MI, Brown-Elliott BA, Wallace RJ Jr, et al. Taxonomic variation in the Mycobacterium fortuitum third biovariant complex: description of Mycobacterium boenickei sp. nov., Mycobacterium houstonense sp. nov., Mycobacterium neworleansense sp. nov. and Mycobacterium brisbanense sp. nov. and recognition of Mycobacterium porcinum from human clinical isolates. Int J Syst Evol Microbiol. 2004;54(Pt 5):1653-67.

5. Levasseur A, Asmar S, Robert C, Drancourt M. Draft genome sequence of Mycobacterium houstonense strain ATCC 49403T. Genome Announc. 2016; 4(3):e00443-16.

6. Clinical and Laboratory Standards Institute. Performance Standards for Antimicrobial Susceptibility Testing, Twenty-eighth Informational Supplement, M100-S28. Wayne, PA: Clin Lab Stand Institute; 2018.

7. Clinical and Laboratory Standards Institute. Interpretive Criteria for Identification of Bacteria and Fungi by DNA Target Sequencing; Approved Guideline , MM18-A. Wayne, PA: Clin Lab Stand Institute; 2008.

8. Lane DJ. 16S/23S rRNA sequencing. In: Stackebrandt E, Goodfellow M, editors. Nucleic acid techniques in bacterial systematics. Chichester: Wiley; 1991. p. 115-75.

9. Clinical and Laboratory Standards Institute. Susceptibility Testing of Mycobacteria, Nocardiae, and Other Aerobic Actinomycetes; Approved Standard- Second Edition M24-A2. Wayne, PA: Clin Lab Stand Institute; 2011

10. Lorencova A, Klanicova B, Makovcova J, Slana I, Vojkovska H, Babak V, Pavlik I, Slany M. Nontuberculous mycobacteria in freshwater fish and fish products intended for human consumption. Foodborne Pathog Dis. 2013;10(6):573-6.

11. Wallace RJ Jr, Swenson JM, Silcox VA, Good RC, Tschen JA, Stone MS, Spectrum of disease due to rapidly growing mycobacteria. Rev Infect Dis. 1983:5(4):657-79.

12. Hamid ME. Current perspectives on Mycobacterium farcinogenes and Mycobacterium senegalense, the causal agents of bovine farcy. Vet Med Int. 2014:2014:247906

13. Nash KA, Andini N, Zhang Y, Brown-Elliott BA, Wallace RJ Jr. Intrinsic macrolide resistance in rapidly growing mycobacteria. Antimicrob Agents Chemother. 2006;50(10):3476-8.

14. Wallace RJ Jr, Swenson JM, Silcox VA, Bullen MG. Treatment of nonpulmonary infections due to Mycobacterium fortuitum and Mycobacterium chelonei on the basis of in vitro susceptibilities. J Infect Dis. 1985;152(3):500-14.

15. Plemmons RM, McAllister CK, Liening DA, Garces MC. Otitis media and mastoiditis due to Mycobacterium fortuitum: case report, review of four cases, and a cautionary note. Clin Infect Dis. 1996;22(6):1105-6.

16. Wallace RJ Jr, Brown BA, Silcox VA, Tsukamura M, Nash DR, Steele LC, Steingrube VA, Smith J, Sumter G, Zhang YS, et al. Clinical disease, drug susceptibility, and biochemical patterns of the unnamed third biovariant complex of Mycobacterium fortuitum. J Infect Dis. 1991;163(3):598-603.

Ready to submit your research? Choose BMC and benefit from

- fast, convenient online submission

- thorough peer review by experienced researchers in your field

- rapid publication on acceptance

- support for research data, including large and complex data types

- gold Open Access which fosters wider collaboration and increased citations

- maximum visibility for your research: over $100 \mathrm{M}$ website views per year

At BMC, research is always in progress.

Learn more biomedcentral.com/submissions 